\title{
INFORMACIONES SOBRE LA REDACCIÓN DE EL SUICIDIO Y EL CONFLICTO ENTRE DURKHEIM Y TARDE DE 1895 A 1897
}

Massimo Borlandi*

Universidad de Turín, Ciencias Políticas

\begin{abstract}
RESUMEN
Durkheim obtuvo algunos de los datos estadísticos sobre el suicidio de Tarde, director de estadística del Ministerio de Justicia de 1894 a 1900, contra cuyas posiciones teóricas El Suicidio construye una de sus críticas más demoledoras. El trabajo estudia las relaciones entre Durkheim y Tarde en el marco de la elaboración de El Suicidio, reconstruyendo su calendario de redacción y analizando la estrategia durkheimiana para obtener datos imprescindibles de quien era un rival en la naciente sociología y centro de sus críticas. Como apéndice aporta unas cartas inéditas de Durkheim a su rival.
\end{abstract}

Emile Durkheim y Gabriel Tarde se conocieron en París el 15 de octubre de 1894, según el Diario de Tarde (Milet, 1970: 33), pero antes de esta fecha ya habían tenido la ocasión de enfrentarse. Durkheim, en su tesis de doctorado, había criticado algunos pasajes de Las leyes de la imitación y de La criminalidad comparada (Durkheim, 1893: 118-119, 204, 286, 329, 420) y había vuelto a tratar del «ingenioso sistema del señor Tarde» en el primero de los cuatro artículos que forman Las reglas del método sociológico (Durkheim, 1894: 473). Tarde, que había contraatacado inmediatamente a La división del trabajo social (Tarde, 1893: 625-632), acababa de pronunciar el 3 de octubre, con oca-

* Durkheim Studies, vol. IV, 1994, pp. 4-13. 
sión del primer Congreso Internacional de Sociología, una vigorosa acusación contra Las reglas (Tarde, 1895d: 212-229, 241) y había entregado a su revista, los Archives d'anthropologie criminelle, un artículo sobre la progresión de la criminalidad en Francia que terminaba con un nuevo ataque contra Durkheim (Tarde, 1894: 650). Su último encuentro tuvo lugar en diciembre de 1903, en la Ecole des Hautes Etudes Sociales, cuando el programa de una serie de conferencias los puso frente a frente en un debate público - muy tenso (Pournin, 1904: 86-87; Guillaume de Tarde, 1904: 20)— que no añadió nada a los términos de una polémica que desde entonces comprometía también a sus partidarios: del lado de Durkheim los grupos compactos del Année sociologique y de Notes critiques; mientras que Tarde gozaba de una solidaridad difusa, sobre todo en el extranjero, a través del Instituto de René Worms. A la muerte de Tarde, el 12 de mayo de 1904, se podían contar 12 textos de Durkheim con críticas de Tarde o redactados contra él, y al menos 14 textos de Tarde total o parcialmente contra Durkheim. Sin contar, en la bibliografía de Tarde, las pequeñas menciones y las numerosas alusiones.

Como sabemos, el texto de Durkheim que más trata de Tarde es El Suicidio. Todo el capítulo IV del libro I, dedicado a la imitación, causa secundaria según Durkheim, principal para Tarde, de la propagación de los fenómenos sociales, incluido el suicidio (30 pp.); el párrafo III del capítulo I del libro III, donde Durkheim responde punto por punto a los comentarios que Tarde le había dirigido anteriormente (18 pp.); y tres páginas del capítulo II del libro III, sobre la disminución del número de homicidios en Francia, cuya evidencia niega Tarde. A lo que hay que añadir el espíritu con el que se concibió la obra: demostrar la debilidad de la explicación individualista de las acciones humanas, precisamente la manera de explicar de Tarde. Tratándose de un tiro al blanco, El Suicidio tiene de particular que Durkheim pudo escribirlo en parte porque Tarde, director de la Estadística judicial francesa entre febrero de 1894 y enero de 1900, le abrió los archivos del Ministerio de Justicia, como se lee al final del prefacio. Aunque no dispusiéramos de este prefacio (que, por otra parte, desapareció desde la segunda edición y que no dice toda la verdad), podríamos deducirlo de las dos ocasiones en que Durkheim emplea datos originales, es decir, no extraídos de las estadísticas oficiales. La primera es de nuevo en el capítulo IV del primer libro: un gran mapa de Francia, que muestra la distribución de los suicidios por distrito de 1887 a 1891 (mientras que los datos oficiales están agrupados por departamento), desmiente, o pretende desmentir, la existencia de focos de irradiación de contagio imitativo. La segunda vez es en el capítulo III del libro II: Durkheim relaciona la edad, el sexo, el estado civil y el lugar de residencia (en el Departamento del Sena o en provincias) de 25.000 fallecidos por suicidio de 1889 a 1891. En este último caso, Durkheim declara el origen de los datos: «documentos inéditos que posee el Ministerio de Justicia» (Suicide, pp. 179-189; ver también pp. 310 y 412), y basta con preguntarse quién estaba a la cabeza de la oficina donde se conservaban los documentos (informes individuales), ya que todos los elementos con- 
textuales excluyen el que Durkheim haya efectuado su investigación antes de 1894. En el primer caso, Durkheim calla sus fuentes, pero son las mismas, y se comprende su silencio: tendría que haber declarado que criticaba a Tarde... con la ayuda de Tarde (lo que efectivamente ocurrió). Después de todo, el capítulo IV del libro I es bastante marginal en El Suicidio. Sin embargo, sólo en este capítulo Durkheim opone hechos, y no meras argumentaciones, a la teoría más famosa de su adversario. Por el contrario, el capítulo III del libro II es completamente central, dada la extensión de la investigación que contribuye a definir un estado de la sociedad - la integración - como una realidad exterior a los individuos que coacciona su conducta; lo que nos lleva a Tarde y a la disputa entre los dos métodos. En resumen, sin la ayuda de Tarde, El Suicidio sería diferente, y mucho menos anti-Tarde de lo que es. Pero ¿cómo es posible que Tarde transmitiera a Durkheim los datos con los que éste lo refutó? ¿Cómo pudo dar pruebas de tal ligereza?

Las cinco cartas de Durkheim a Tarde que publicamos en anexo de esta nota, y que constituyen su razón de ser — cuatro de 1895 y una de 1897, todas inéditas a excepción de ocho líneas de la primera (ver Milet, 1973: 24)—, nos permiten conocer mejor esta cuestión. Contienen informaciones preciosas para determinar la fecha (o fechas) de la redacción de El Suicidio y también para determinar las fuentes estadísticas que Durkheim utilizó o no. Por último, la actitud de Durkheim respecto a Tarde se aclara un poco más.

Todo parece comenzar con la Exposición internacional de Burdeos en 1895: 11 de mayo-17 de noviembre. Durkheim participa, ya que incluso los resultados de la sociología (¡maravillas de la época del cientismo!) se exponen. El episodio es oscuro aunque quedan huellas: una reminiscencia de Marcel Mauss (1925: 14) recordando que Durkheim elaboró para la ocasión un cuadro "filogenético", es decir, evolutivo, de la organización doméstica; y un informe de la universidad de Burdeos, también de 1895, señalando que «el señor Durkheim (...) se ha ocupado activamente de la Exposición de ciencias sociales y ha figurado con importantes trabajos de estadística, entre otros un curioso mapa del suicidio en Francia». Este extracto debe interesarnos. Separemos ambas cosas, los trabajos de estadística y el mapa curioso. El tema de los primeros no es difícil de imaginar. Hasta entonces, Durkheim sólo ha tratado en sus cursos de dos temas a la luz de la estadística: el suicidio (1889-1890) y la criminalidad (1892-1893 y 1893-1894). Su único texto acompañado de datos estadísticos trata del suicidio en relación con la natalidad (Durkheim, 1888). Su familiaridad con las cifras de la criminalidad resulta de Las reglas y de su respuesta, "Crimen y salud social», al artículo de Tarde casi homónimo (cuarto vs. Durkheim, tercero a propósito de Las reglas) ${ }^{1}$. Además, Durkheim revela en esta respuesta —estamos en mayo, pero el texto está fechado en marzo (carta 1)— que está trabajando en El Suicidio (Durkheim, 1895c: 518).

Pero, se trate de la criminalidad o bien del suicidio, nada cambia. En todo

${ }^{1}$ G. Tarde (1895b). 
caso, Durkheim sólo puede haber presentado o elaborado en Burdeos, respecto a Francia, datos publicados en el Compte général de l'administration de la justice criminelle, la serie anual que pone al día la variación de ambos fenómenos desde 1826 (la del suicidio en la división séptima, titulada «Informaciones diversas»). Entonces — cartas 1 y 2 (25 y 31 de marzo)—, Durkheim pidió a Tarde dos volúmenes de esta serie del Ministerio de Justicia ${ }^{2}$ expresamente para exponerlos en Burdeos. Los obtuvo, no sin dificultad. Se comprometió a darles la máxima visibilidad (la "pregunta" previa de Tarde parece limitarse a esto), precisando que haría un uso personal, es decir, que los utilizaría para o durante la exposición (probablemente una o varias conferencias) de sus doctrinas (lo que podría llamarse la exposición de sí mismo y confirma el informe de la universidad: Durkheim se reservó en la Exposición internacional un lugar para sí). La carta 4 (15 de noviembre), donde el Compte général es llamado Compterendu ${ }^{3}$, da finalmente las referencias de estos dos volúmenes: 1882 y 1887. ¿Qué hace Durkheim concretamente de ellos? ¿Los consulta? ¿Saca fichas? Lo que significaría que nunca los había visto. ¿O los utiliza tal cual, únicamente como material de soporte para enseñar, como será el caso, veinte años después, de las 14 obras de sociología que figuran en la Biblioteca de la ciencia francesa en la Exposición universal de San Francisco (ver Durkheim, 1915: 48-49)? El hecho de que Durkheim devuelva o quiera devolver a Tarde los dos volúmenes (carta 4) no aclara la cuestión: en efecto, podría habérselos devuelto tras haber recopiado lo que le interesaba. $\mathrm{Ni}$ puede sernos de ayuda el suponer que difícilmente Durkheim pudo haber dado de 1892 a 1894 dos cursos de sociología criminal —en profundidad, al parecer ${ }^{4}$ — sin haber tratado el movimiento de la criminalidad en Francia durante los años ochenta. Efectivamente, Durkheim pudo haber desarrollado este tema gracias a los textos de otros autores. ¿No está El Suicidio, en más de su mitad, así construido?

Volvamos al mapa curioso. No hay duda, se trata del gran mapa del capítulo IV del libro I de El Suicidio (pp. 124-125): «Tabla II. —Suicidios en Francia, por distritos (1887-1891)». La anomalía de este mapa radica en su originalidad misma. ¿Es concebible que Durkheim se haya esforzado en establecer hasta los más pequeños detalles de la geografía del suicidio en Francia y que no hable de ello en su libro? Veamos la carta 3 - una nota de presentación con fecha del mes de junio o julio y que deja entrever una visita de Durkheim a Tarde 5 - un «estudiante de buena voluntad» va al Ministerio de Justicia para

${ }^{2}$ Como puede leerse en el sobre de la carta 2, uno de los dos conservados (el otro es de la carta 3).

${ }^{3}$ Ver la misma expresión en El Suicidio (pp. XII, 16 y 286). Es una expresión corriente (Tarde, 1886: 63).

${ }^{4}$ En una comunicación a Henri Hauser, Durkheim precisará que esos cursos trataban de los "principales tipos de criminalidad», cuyas «condiciones históricas y demográficas» había estudiado (Hauser, 1903: 168).

5 Nuestra datación se basa en los hechos siguientes. Durkheim está en París, lo que hace pensar en las vacaciones escolares. Pero difícilmente pueden ser las vacaciones de primavera (Pas- 
que Tarde le dé «la cifra anual de suicidios por distrito para los cinco años más recientes» de que dispone. En efecto, la tabla II de El Suicidio cubre cinco años, el último 1891, ya que a partir de 1892 la documentación de los suicidios no está centralizada en París (ver Besnard, 1987: 77). Lo que significa, dada la única utilización posible de este mapa, que en Burdeos, durante la Exposición internacional, Durkheim adoptó públicamente una posición contra las tesis de Tarde. Ahora bien, como es improbable que Durkheim haya advertido a Tarde de sus intenciones, debemos tener en cuenta la sutil y objetiva perfidia que se esconde bajo las palabras de la carta 3: ese "pequeño trabajo» que Tarde consiente en realizar va a convertirse en la base empírica de la crítica más aguda que haya recibido en su vida. De todas formas, aun suponiendo que el mapa curioso no haya sido preparado desde el principio para El Suicidio, se encuentra de hecho en este texto. Los compromisos con la Exposición y la preparación del libro se cruzan; de manera que, una vez terminada la Exposición, la investigación continúa. Y en noviembre (carta 4) nos encontramos a Mauss («mi sobrino»), que acaba de obtener la agregación, bien instalado en el despacho de Tarde (que ya no desconfía). ¿Qué hace allí?

El prefacio de El Suicidio lo dice, y Mauss mismo lo escribirá en un curriculum vitae de 1930 (Mauss, 1979: 210): se encarga de analizar los 25.000 informes individuales de los que habla el capítulo III del libro II ${ }^{6}$. Por el contrario, el prefacio no menciona el otro favor de Tarde. Durkheim da las gracias a cierto "señor Ferrand, profesor de la Escuela primaria superior de Burdeos», también "antiguo alumno" suyo, por haber hecho los mapas del libro (10 en total, pero en realidad ocho ya que el primero se reproduce tres veces). ¿Hay que leer entre líneas que para elaborar el quinto mapa, el de suicidios por distrito, el señor Ferrand debió ir a París, y que él y ese estudiante de buena voluntad del que habla la carta 3 son una sola y única persona? Si no fuera así, ¿cómo Durkheim habría podido olvidar dar las gracias a este primer emisario?

El listado del número de suicidios por distrito necesita - en nuestra opinión- cerca de una semana. En el fondo, se trata de recopiar los datos de 1.810 cuadros estadísticos redactados por los fiscales de la República ${ }^{7}$. Obtene-

cua en 1895 cae el 14 de abril): en efecto, ¿por qué Durkheim solicitaría, a fines de marzo, el envío de dos volúmenes de estadísticas si sabe que puede cogerlos él mismo diez o quince días más tarde? Además, normalmente, Tarde se va de París a La Roque Gajac durante las vacaciones. Así que sólo nos queda el verano. Excluyendo el período entre fines de julio y primeros de septiembre, la alternativa queda entre las semanas a caballo entre junio-julio y después de la vuelta de vacaciones. Pero ¿queda tiempo, a partir de mediados de septiembre, de hacer la lista para el Ministerio de Justicia, de preparar el mapa y de presentarlo en Burdeos antes del cierre de la Exposición a mediados de noviembre? Teóricamente, sí (como lo diremos seguidamente), pero es improbable.

${ }^{6}$ Mauss dice, en verdad, 26.000. Durkheim, que escribe 25.000 («alrededor de») en el libro II, también dice 26.000 («alrededor de») en el prefacio. Ver Besnard (1987: 76-77) sobre la credibilidad de estas cifras.

${ }^{7}$ Ver en el Curso de Jacques Bertillon (1895: 285-286) cómo está organizado el servicio de la estadística judicial. 
mos esta cifra multiplicando 362 (los distritos) por 5 (los años). En cambio, Mauss sólo pudo acabar su trabajo después de varios meses. Admitiendo que llegara a analizar hasta 300 informes al día, haciendo un cálculo optimista (también señala la presencia de niños), si comienza en noviembre (carta 4), aún es el "huésped» de Tarde en enero, incluso en febrero de 1896. La redacción de El Suicidio se ha fechado hasta ahora en 1895 , entre la primavera y el otoño, para el libro I y el libro II, hasta el capítulo IV, inclusive; y en 1896, del mes de mayo hasta el primer o segundo mes de 1897 , en relación a los capítulos V y VI del libro III (Besnard, 1987: 130-133), basándose en índices bibliográficos (cuatro si precisamos), en los ejemplos lingüísticos y temáticos de asincronismo, así como en una neta concordancia (respecto del tema de la anomia, capítulo $\mathrm{V}$ del libro II) con la decimosegunda lección (una de las últimas) del curso sobre la historia del socialismo impartido por Durkheim en 18951896. Según esta hipótesis, la causa de la interrupción (noviembre 1895-abril 1896) sería este mismo curso (ibid.) Las cartas a Tarde confirman que Durkheim comienza a redactar El Suicidio (al menos la redacción final) en 1895, pero sugieren que la pausa (si fue tan larga) tuvo lugar entre los capítulos II y III, y no entre los capítulos IV y $\mathrm{V}$ del libro II. En efecto, la redacción del capítulo III del libro II presupone haber terminado el análisis de los 25.000 informes y, además, tener resultados bien legibles (los cuadros XXI y XXII de pp. 183 y 204). A su vez, el capítulo siguiente (el IV, sobre el suicidio altruista) utiliza datos procedentes del análisis de Mauss (ver pp. 249, 253-254 y 258). Así, Durkheim no se aplica al capítulo III del libro II, como al resto de El Suicidio, antes de febrero-marzo 1896. Y cabría preguntarse también si la lentitud del trabajo de Mauss en el Ministerio de Justicia no ha sido una de las causas del paréntesis en la redacción de la obra. En relación a la otra causa, más concomitante que principal, nos maravillamos de nuevo de la abnegación de Durkheim. Agobiado por una pesada tarea cuyo fin no llega a ver, decide, sin embargo, comprometerse a preparar un curso que le obliga a nuevas lecturas detalladas (Sismondi, Saint-Simon y toda una literatura secundaria) en vez de volver a impartir un curso ya redactado, como hará cuando se ocupe del Année. Pero la única prueba (hasta ahora) de que el curso sobre el socialismo data de 1895-1896 esta ahí, indiscutible: Annuaire des facultés de Bordeaux, 1895 , p. 116. (aunque es verdad que a veces los profesores, obligados a comunicar con mucha antelación el tema de sus cursos, lo cambian al comienzo del año universitario) $)^{8}$.

Pero volvamos a los dos volúmenes de estadística enviados por Tarde a Durkheim para la Exposición de Burdeos. Que Durkheim los conociera ya o nos los hubiera nunca visto, el hecho es que los encontramos en El Suicidio. En efecto, Durkheim sólo cita en el libro tres volúmenes del Compte général de l'administration de la justice criminelle, y dos de esos volúmenes son los de

8 Señalamos de paso que Mauss fechó una vez en 1897-98 el curso sobre el socialismo (Mauss, 1925: 15). 
1882 y 1887 (pp. 46 y 166). Un hecho notable, ya que Durkheim sigue la regla de no nombrar nunca sus fuentes estadísticas oficiales. Se esconde tras la lista que da al final de la introducción, un tanto desordenada y sin precisar las fechas; pero hace una excepción con esos dos volúmenes. Además, el año 1887 — segundo volumen - es el último de la década sobre la que Durkheim trabaja. Se trata del porcentaje departamental de suicidios (N/ 100 000) calculado para la década 1878-1887, que entra en las grandes comparaciones de las tablas I, IV y V (suicidio y, respectivamente, alcoholismo, densidad familiar, riqueza). A esta década se refieren los datos (comparados) sobre la profesión de los suicidas (p. 286) y los de los homicidios del capítulo II del libro III (el año 1887 siempre en evidencia, p. 390). No podemos equivocarnos: El Suicidio dedica un lugar importante a los dos volúmenes del Compte général cuyo ejemplar Durkheim pidió a Tarde en el mes de marzo de 1895. Ciertamente, junto a otros volúmenes, pero no muchos más; por ejemplo, no los de 1878 y 1879 . ¿Por qué no estos últimos? Porque para los años que preceden a 1881, Durkheim se apoya, aunque de manera poco sistemática, en el volumen relativo a 1880 , que ya había utilizado en su artículo sobre el suicidio y la natalidad (Durkheim, 1888: 450) y que es, en efecto, el tercer volumen citado del Compte général (p. 286). Este volumen se caracteriza por estar precedido de un informe de recapitulación que abarca medio siglo (1830-1880). La falta de claridad con la que Durkheim se sirve de este volumen radica en que, al mismo tiempo y para los mismos años, saquea los libros de Alfred Legoyt (1881), Armand Corre (1891), naturalmente el de Enrico Morselli (1879) y también otros. Habiendo aclarado esto, el problema se plantea para los años posteriores a 1887. Cuando se publicó El Suicidio hubo quien señaló que sus datos no eran de lo más reciente. Augusto Bosco (1897: 380) se limitó a una alusión, pero Vilfredo Pareto asestó un golpe más duro y escribió que Durkheim había descuidado (¿deliberadamente?) las últimas estadísticas francesas de suicidio. Citaba en particular una que parecía conocer bien: la del Compte général de 1893 (Pareto, 1898: 79). Pareto buscó la prueba de esta omisión donde no habría debido, en los cuadros I y II de la introducción (su lectura fue apresurada y sesgada), pero ahí están los hechos: después del volumen de 1887 y antes de que El Suicidio fuera entregado al editor, seis volúmenes más del Compte général fueron publicados. El de 1888 en 1891, con un retraso del que el mismo Tarde (1891: 325) se quejó. Los de 1889 y 1890 en 1893. El de 1891 en 1894. El de 1892 en 1895. Y el último, precisamente del que habla Pareto, en 1896. Pero no a finales de año, sino más bien al comienzo. Prueba de ello es que Tarde lo cita varias veces en un texto, fechado en mayo, presentado en agosto al Cuarto Congreso Iinternacional de Antropología Criminal (Tarde, 1896b: 539, 546-548) . Ahora bien, es importante saber si Durkheim utilizó o

9 Es uno de los dos congresos de que habla Durkheim en la carta 2 (Ginebra, 24-28 de agosto de 1896). El otro es el Quinto Congreso Penitenciario Internacional (París, 29 de junio-12 de julio de 1895). 
no en su libro estos seis volúmenes, cuáles y en qué medida. Cuanto menos se sirve de ellos, más antiguo es El Suicidio. No en cuanto a su redacción material (al menos la redacción final), para la que no se puede remontar a antes de 1895; pero desde hacía tiempo las grandes líneas del libro estaban establecidas, y sobre una base estadística suficiente, en un trabajo preparatorio (y por supuesto en la cabeza) de su autor. Miremos El Suicidio más de cerca.

Encontramos pocos datos sobre el suicidio en Francia en 1888. Son numerosos para los años 1889, 1890 y 1891, pero hay que separar los que proceden del listado del Ministerio de Justicia, lo que no siempre es fácil. Además, aunque pueda parecer irrespetuoso, se debe desconfiar de todo el aparato estadístico de El Suicidio, ya que Durkheim, las más de las veces, olvida citar las cifras absolutas. Además, hay un 1892 («-92») en una nota de pie de la página 295 que era claramente 1891 en el manuscrito. Los pocos datos del año 1888 se limitan seis de cada ocho veces a cifras proporcionales medias (por ejemplo, p. 193: «durante los años 1887-1891, un millón de esposos sin hijos dio anualmente 644 suicidios»; ver también pp. 190, 219, 247, 295, 301) o desaparecen bajo un porcentaje (p. 420: la agravación del suicidio en Francia fue de 385 por 100 «de 1826 a 1888»). Solamente una vez, p. 217, vemos datos absolutos. Lo mismo podemos decir de los datos de 1889-1991, que no parecen proceder directamente del listado de Mauss. Seis de cada siete veces (con el mismo motivo que los datos de 1888) contribuyen a formar una cifra proporcional media. Sólo una vez, siempre en la p. 217 , se presentan en cifras absolutas, excluido 1891. La p. 217 propone un pequeño cuadro, además bien cono$\operatorname{cido}^{10}$, donde figura, mes por mes, de mayo a diciembre, el número de suicidios de 1876 a 1878 y de 1888 a 1890. Ahora bien, Durkheim sólo puede haber obtenido del Compte général los datos de este cuadro para 1888. Efectivamente, ni siquiera el libro de Corre (1895) cita estos datos, que sólo fueron publicados, como acabamos de decir, en 1891. También obtuvo Durkheim del Compte général los datos para 1889 y 1890 de este mismo cuadro. Tanto más seguro cuanto que Mauss no hizo un listado sobre las variaciones mensuales de los suicidios. Esto es todo lo concerniente a Francia después de 1887 —respecto de los datos oficiales, exceptuando la ciudad de París ${ }^{11}$ - . Lo que significa que de los seis volúmenes de estadísticas oficiales disponibles - cinco (18881892) ya en 1895-, Durkheim utilizó ciertamente los cuatro primeros (en particular los tres primeros), pero casi para nada. El Suicidio (junio de 1897) dista diez años largos del grueso de sus fuentes estadísticas francesas. Es cierto que, sin ser su función, los datos del análisis de Mauss reducen esa separación. Prueba de ello es la imposibilidad de ligarlos a las series históricas (o de anexarlos a las comparaciones) que estructuran el resto de la obra. Hay que añadir

${ }^{10}$ El golpe de estado parlamentario de mayo de 1877 y las elecciones de septiembre 1889 (fin del boulangismo) forman parte de las pruebas de que las crisis políticas causan una baja de los suicidios.

${ }^{11}$ Para los suicidios cometidos en París, Durkheim llega hasta 1892 (El Suicidio, p. 105). Su fuente es el Annuaire statistique. 
que rara vez las estadísticas de otros países europeos considerados en El Suicidio van más allá de 1890. El conjunto de estos datos se concentra sobre todo en los años sesenta y setenta del siglo XIX.

Una observación y una pregunta pueden ayudarnos. Primero, la observación: los pocos datos oficiales franceses sobre los años 1888-1891 se encuentran en el capítulo III del libro II o en los capítulos siguientes. Han sido añadidos después de la interrupción del invierno 1895-1896. A continuación, la pregunta: ¿por qué Durkheim elige, en 1895, trabajar sobre los años 18781887, en vez de, por ejemplo, sobre los años 1880-1889 ó 1882-1891 (puede llegar hasta 1892)? La elección de una década más cercana hubiera dado mucho más valor a las comparaciones que Durkheim estableció con los datos del recuento de 1886. Sólo hay una respuesta posible: en 1895, Durkheim posee ya, probablemente bien ordenados en cuadros, una buena parte de su documentación estadística. Le conviene, en previsión de una conclusión rápida de su obra, completarla sin dispersarse demasiado. Y como hay que terminar esa «investigación» suplementaria sobre la redacción de El Suicidio (Besnard, 1987: 130) debida al descubrimiento de un extraño viaje de París a Burdeos (y vuelta probable) de dos volúmenes de estadísticas en 1895, formulamos las hipótesis siguientes. El punto de partida y el primer esbozo de El Suicidio es el texto del curso de 1889-1890. En este curso hay varios datos que Durkheim extrae de la "estadística moral» del siglo XIX (todos los estudios, salvo dos, utilizados en El Suicidio fueron publicados antes de 1887), así como los datos oficiales franceses que preceden a 1881; también se pueden encontrar datos oficiales de otros países. En 1895, después de Las reglas y con la finalidad de dar ejemplos de su método, Durkheim decide publicar su curso. Entonces integra su documentación original, sobre todo junto a las estadísticas oficiales. Pero sucede algo inesperado y, a fin de cuentas, favorable (al menos para la durkheimiología del siglo Xx): Tarde, adversario también inesperado, pero amable, ofrece a Durkheim la posibilidad de repetir la experiencia de Alexandre Brierre de Boismont, que había analizado los informes de 4.595 suicidios cometidos en París entre 1834 y 1843 (El Suicidio, p. 98). En ese momento todo se para y se enreda. Y, cuando vuelve a la redacción del libro, en 1896, Durkheim ya no tiene tiempo y, probablemente, ni siquiera interés en reorganizar las estadísticas; aún menos la voluntad de abrir este último volumen del Compte général de l'administration de la justice criminelle recién impreso que trata de 1893. El Année y muchas otras cosas urgen.

El trato que Durkheim y Tarde establecen en 1895, aunque a distancia y por intermediarios ${ }^{12}$, no reduce el conflicto que los opone. Al contrario, para Durkheim, no es más que el principio. Los dos textos que Durkheim publica sobre Tarde en el mes de mayo de ese año: «Crimen y salud social» y el párrafo II del artículo sobre la sociología francesa redactado para una revista italiana

${ }_{12}$ Pero antes de la llegada de Mauss al Ministerio de Justicia (noviembre), o bien otro intercambio de cartas tuvo lugar, o Durkheim y Tarde se volvieron a ver. 
(Durkheim, 1895b: 615-622), deben ordenarse según una sucesión correcta. El segundo texto, que aún muestra un ligero aprecio por la obra de Tarde, fue escrito antes que el primero, es decir, mucho antes que Durkheim tuviera la impresión de que Tarde estaba haciéndole la guerra. Y, lo que es más, ambos textos preceden la publicación, en el primer volumen de los Annales de l'Institut international de sociologie, del informe presentado por Tarde en el Congreso de octubre de 1894 que contiene, como hemos dicho, un análisis (corrosivo) de Las reglas. La lectura de este informe persuade a Durkheim de que, a pesar de los favores que Tarde le hace en el Ministerio de Justicia, no debe dudar en atacarle: el párrafo III del capítulo I del libro III de El Suicidio (el más teórico del libro) está enteramente construido contra el texto de Tarde de octubre de 1894. Por otra parte, en 1895 se define la imagen de Durkheim y Tarde como polos intelectuales opuestos. Durkheim se da cuenta a partir de las reacciones a Las reglas. Georges Sorel, que trata de Las reglas en el Devenir social, va a contarles a los italianos (él también, pero en francés) los puntos de vista de Durkheim y de Tarde sobre el crimen y la pena (Sorel, 1895 ${ }^{13}$ ). Paul Lapie (1895: 314), futuro colaborador, recalca mucho más de lo necesario el corto pasaje que Tarde dedica a Durkheim en el prefacio de su Lógica social (Tarde, 1895a: VII-VIII $\left.{ }^{14}\right)$; y Lucien Lévy-Bruhl (1895) hace una reseña de Las reglas donde habla igualmente de otros libros de sociología, entre ellos los de Tarde. Lo que no alegra a Durkheim, quien sin embargo felicita a Lévy-Bruhl por haber, al menos, explicado bien su método: «Le estoy particularmente agradecido por haber dicho que este método (...) tiene al menos la ventaja de poner una barrera entre los trabajadores serios y los embaucadores o los diletantes» (Durkheim, 1895d). El término «embaucadores» (faiseurs), con el que Durkheim alude a Tarde (¿a quién si no?, ¿no se convertirá en una contraseña del grupo del Année decir que Tarde improvisa o, a lo sumo, escribe literatura?), se encuentra en el artículo de Lévy-Bruhl, aunque no haya una referencia directa; pero podemos leer también este término en la carta de Durkheim a Tarde del 31 de marzo. Tras haber anticipado a Tarde el contenido de "Crimen y salud social» (carta 1), Durkheim no encuentra nada mejor, para engatusarlo y continuar el deshielo, que decirle que, en definitiva, a pesar de todo lo que les separa, ambos están del mismo lado contra «la invasión de los embaucadores en la sociología» (carta 2). Es totalmente normal que Durkheim, al necesitar la ayuda de Tarde, intente minimizar sus discrepancias. Lo que sorprende es su rencor en aumento contra Tarde desde 1895. Un rencor que no se detendrá ni siquiera tras la publicación de El Suicidio. (Y todavía estamos lejos de los acontecimientos de 1900, cuando Tarde, en la cumbre de su éxito mundano y parisino, será nombrado al Colegio de Francia, mientras que Durkheim queda relegado en su exilio de Burdeos.)

13 Sorel comparte las tesis de Durkheim. Tarde (1896a: 644) parece estar al corriente de este artículo.

${ }^{14}$ Este libro será publicado en 1994. 
A fines de septiembre de 1897, Durkheim devuelve a Celestin Bouglé el manuscrito de los análisis que éste acaba de preparar para la primera sección (sociología general) del primer volumen del Année. La carta adjunta la toma con la reseña de la Philosophie des Geschichte als Soziologie de Paul Barth. Bouglé se queja de que éste no haya considerado suficientemente a Tarde, al que califica de «representante de la sociología específica». ¿No sería algo exagerado?, se pregunta Durkheim. Tarde niega la especificidad de los hechos sociales, es decir, que el todo sea diferente de la suma de sus partes, o que la sociología sea diferente de la psicología individual; por lo tanto, no merece la pena dar una importancia tan grande a la omisión de Barth (Durkheim, 1897: 409). ¿Qué libro de Tarde se cuestiona? Naturalmente, una vez más, el texto de octubre de 1894: "Todo esto está dicho formalmente en un trabajo, dirigido contra mí, y publicado en los Annales de Worms» (ibid.). Hay que señalar que Bouglé, que parece corregir su texto, lo hace preceder de una severa reseña de la Oposición universal (Bouglé, 1898a, b). En este contexto hay que situar la carta de Durkheim a Tarde del 16 de julio de 1897. Durkheim envió El Suicidio a Tarde (según podemos imaginar, con una dedicatoria respetuosa). Tarde le comunicó su intención de rebatirle; pero, a decir verdad, debe haberle hablado sobre todo de otras cuestiones: de pura casualidad, su hijo mayor, Paul, es un alumno de Durkheim en Burdeos (carta 5). De todas maneras, a propósito de la réplica, Durkheim parece casi esconderse, lo que nos pone al límite del engaño. Dice desinteresarse de las cuestiones teóricas que Tarde quiere plantear de nuevo: "Dejo de lado cada vez más esos problemas generales» (ibid.). Es decir, los problemas generales que había minuciosamente tratado en el capítulo I del libro III de El Suicidio; esos mismos problemas a los que va a aferrarse, contra Tarde, en la carta a Bouglé que acabamos de citar (el todo, la parte, lo social y lo individual). La espera de esta réplica pone nervioso a Durkheim. En la primavera de 1898 aún no ha llegado. Durkheim, al enviar a Xavier Léon, para la Revue de métaphysique et de morale, su artículo sobre las representaciones individuales y colectivas (problemas generales), le pide que lo publique lo más rápido posible: quiere evitar que ese artículo, que corre el riesgo de publicarse tras el «ataque» con el que Tarde le ha amenazado, pueda parecer una respuesta. Está decidido a parar ese debate que "ha durado demasiado" (Durkheim, 1898: 464; ver también Lukes, 1973: 303-304).

La réplica de Tarde tarda en llegar y, en definitiva, no llega. Salvo que consideremos como tal las cinco líneas del apéndice añadido por Tarde a la nueva edición, en 1898, del artículo de 1895 ("Criminalidad y salud social») que había provocado "Crimen y salud social». Criticando la primacía que Durkheim atribuye a la ciencia y que él siempre ha cuestionado («el origen de nuestra disputa», dijo Durkheim, 1895c: 523), Tarde escribe: «Hasta el conocimiento más objetivo, la estadística, se presta a las interpretaciones más subjetivas, como el reciente libro — por lo demás muy interesante y tan concienzudamente exhaustivo- del señor D[urkheim] sobre el suicidio es la prueba concluyente» (Tarde, 1898: 161). Además, en la revista de Tarde, Camille Tour- 
nier (1898) publica una reseña muy benévola de El Suicidio. Ya que se trata de debatir el capítulo sobre la imitación, lo que necesitaría una investigación apropiada, y, digámoslo, Tarde no se siente verdaderamente cómodo con el análisis de datos estadísticos (no se conoce, en sus trabajos, un solo cuadro de comparación de las variaciones de dos fenómenos). Tratándose de cuestiones teóricas (los "problemas generales») prefiere acechar a Durkheim a la espera de ocasiones mejores. En el fondo, Durkheim continuará escribiendo sobre Tarde y, a pesar de lo que se había propuesto, se verá arrastrado al duelo por su adversario - mucho antes de diciembre de 1903 (Tarde, 1901: 460; Durkheim, 1901).

\section{ANEXO: CARTAS DE EMILIO DURKHEIM A GABRIEL TARDE ${ }^{15}$}

\section{Carta 1}

Burdeos, 25 de marzo de 1895

\section{Estimado señor:}

Me pareció entender en su última carta que me enviaría al mismo tiempo, o que iba a enviarme, los dos volúmenes de estadística que tiene a bien confiarnos; y esperaba la llegada del envío para darle el acuse de recibo y responderle a la vez. Pero, al no ver llegar nada, concluyo que me he equivocado y que usted espera por su parte una respuesta a su pregunta.

Las cosas podrán arreglarse, creo, como usted lo desea. He consultado al señor Samazeuilh, representante de la Sociedad Filomática en nuestro grupo, y no ve ningún inconveniente en que yo exponga los dos volúmenes por mi cuenta. Así que puede enviármelos cuando guste.

Mi respuesta a su artículo es corta porque el señor Ribot me ha aconsejado no hacerla larga para que pueda publicarse sin demasiada demora, y también porque me parece que el autor debe utilizar el derecho de respuesta con moderación. Verá por esta nota que, como usted, juzgo falsas numerosas proposiciones que combate atribuyéndomelas. Me alegraría que estas explicaciones puedan servir para disminuir la distancia que nos separa; pero el final de su artículo me hace temer que, en un punto esencial, nuestra disensión sea profunda. Le agradezco que me haga saber que este desacuerdo doctrinal no disminuye la estima con la que me honra; no necesito decirle que, por mi parte, la deferencia por su obra y por usted sigue entera. Además, por muy militante que yo sea, soy lo bastante filósofo como para comprender todo el interés que hay en

15 Damos las gracias a la señora de Paul-Henri Bergeret, de soltera Françoise de Tarde, nieta de Gabriel, que ha tenido la amabilidad de autorizarnos a publicar estas cartas. Philippe Besnard nos ha ayudado a elucidar ciertos pasajes y nos ha transmitido amablemente el extracto del informe de la universidad de Burdeos que certifica la participación de Durkheim a la Exposición internacional de 1895 . 
que puntos de vista distintos del mío estén representados en la ciencia, y sin dejar de luchar, me doy cuenta de la utilidad de las resistencias que combato.

Le saluda atentamente.

\section{Carta 2}

Burdeos, 31 de marzo de 1895

\section{Estimado señor:}

He recibido los dos volúmenes de estadística que ha tenido la bondad de confiarnos y le doy las gracias. Los expondremos de manera que puedan ser libremente consultados por nuestros investigadores.

Por lo que se refiere al congreso, me parece que el verdadero favor que podría hacerle al señor $\mathrm{V}[\dot{i} . . . ?]$ sería darle los nombres de los miembros de la organización, tanto del Congreso penitenciario como del Congreso de criminología, si al menos[¿...?] en esta organización permanente. Ya le he advertido además de las pocas posibilidades de éxito de su proyecto, a mi parecer; pero no podía asumir la responsabilidad de comprometerlo formalmente a renunciar. Por esto, pensando que usted es miembro de la organización, le he aconsejado dirigirse a usted.

Hace tiempo que me preocupa la invasión de embaucadores en la sociología. Hace más de dos años que, durante una entrevista con el señor Liard, llamé su atención sobre este peligro que comenzaba a declararse. El mal ha progresado singularmente desde entonces. Creo que el único medio de detenerlo es no siendo en absoluto complaciente con esos sociólogos improvisados que comprometen una ciencia, demasiado fácil ya de comprometer. La desgracia es que todo el mundo se crea así competente; lo que permite, a los que son hábiles, engañar al público. Me alegro de ver que usted siente igualmente el peligro.

Le saluda atentamente.

\section{Carta 3}

París, 162 faub. St. Martin [junio-julio de 1895]

Estimado señor:

Tengo el honor de presentarle, como me ha autorizado, al estudiante de buena voluntad que se encarga del pequeño trabajo del que le he hablado. Le estaría muy agradecido si a usted le pareciera bien comunicarle la cantidad anual de suicidios por distrito para los cinco años más recientes de que disponga.

Dándole las gracias por adelantado, le saluda atentamente. 


\section{Carta 4}

15 de noviembre de 1895

179, Boulevard de Talence, Burdeos

Estimado señor:

Mi sobrino que es su huésped por el momento me ha informado de su vuelta a París. Me he alegrado de saber así que de ahora en adelante ya no está preocupado por la salud de su joven enfermo. Además, el señor M.[¿̇...?] ya me había dado, en octubre, noticias tranquilizadoras.

Mi sobrino me ha hablado también de la benevolencia con la que le ha acogido y tengo el deber de agradecérselo... sin más demora. Temo que abuse de su amabilidad. Lo lamentaría... y también él mismo... si su presencia fuera una molestia para usted. El menor rincón será suficiente para su trabajo: así se alegrará de no tener que preguntarse más si no le estorba en su trabajo.

La Exposición de Burdeos está en vísperas de cerrar sus puertas; el domingo tendrá lugar el cierre definitivo.

A propósito de esto, ¿querría hacerme saber, por favor, lo que debo hacer con los dos ejemplares del Compte-rendu que me confió (años 1882 y 1887)? ¿Puedo devolvérselos vía el tribunal de justicia? No merece la pena que me responda. Bastará con darle sus instrucciones a mi sobrino que me las transmitirá.

Le saluda atentamente.

\section{Carta 5}

16 de julio de 1897

\section{8, Boulevard de Talence, Burdeos}

\section{Estimado señor:}

Pienso que estará muy preocupado por la suerte de su hijo, cuya presencia entre nuestros candidatos ha tenido la discreción de silenciar; es para mí un placer anunciarle que su examen, que acabo de corregir, es bueno. Pienso ponerle 13 o incluso $14^{*}$, lo que multiplicado por el coeficiente 2 le dará desde ahora una ventaja de siete $\mathrm{u}$ ocho puntos.

Encuentro del todo natural que usted me responda, lo que será para mí un honor. En lo que a mí respecta, a no ser que su argumentación lo haga absolutamente necesario, estoy bien decidido a no debatir y a no volver sobre la cuestión, al menos directamente. En primer lugar, no había ninguna razón para que se acabe, y además dejo de lado cada vez más los problemas generales. De

\footnotetext{
* En el sistema educativo francés la puntuación de los exámenes va de 1 a 20 (N. del T.).
} 
ahora en adelante, se juzgará el método por las aplicaciones que he hecho (y que me lo han inspirado en parte), a medida que la enseñanza me vaya dejando el tiempo libre necesario para publicar los resultados obtenidos.

Me alegro mucho de la buena impresión que le ha hecho mi alumno. Respecto del incidente reciente, o más bien inminente, al que usted hace alusión... prefiero no decir nada.

Atentamente le saluda.

\section{BIBLIOGRAFÍA}

Bertillon, J. (1895): Cours élémentaire de statistique, París: Société d'éditions scientifiques.

Besnard, Ph. (1897): L'anomie, París: PUF.

Bosco, A. (1897): «C. r. de E. Durkheim, Le suicide», Rivista italiana de sociologia, 1: 376-383.

Bougle, C. (1898a): «C. r. de G. Tarde, L'opposition universelle», L'AAnnée sociologique, 1: 111116.

- (1898b): "C. r. de P. Barth, Die Philosophie der Geschichte als Soziologie», L'Année sociologique, 1: 116-123.

CORRe, A. (1891): Crime et suicide, París: Doin.

Durkheim, E. (1888): «Suicide et natalité», Revue philosophique, 26: 446-463. [Repr. en Durkheim (1975), 2: 216-236.]

- (1893): De la division du travail social, París: Alcan.

- (1894): «Les règles de la méthode sociologique», Revue philosophique, 37: 465-498. [Repr. en Durkheim (1895a): 1-58.]

- (1895a): Les règles de la méthode sociologique, París: Alcan.

- (1895b): "Lo stato attuale degli studi sociologici in Francia», La riforma sociale, 3: 607-622, 691-707. [Trad. en Durkheim (1975), 1: 73-108.]

- (1895c): «Crime et santé sociale», Revue philosophique, 39: 518-523. [Repr. en Durkheim (1975), 2: 173-180.]

- (1895d): «Lettre à Lucien Lévy-Bruhl (24 juin)», en Merllie (1989: 510).

- (1897): «Lettre à Celestin Bouglé (27 septembre)», en Durkheim (1975, 2: 409-412).

- (1898): «Lettre à Xavier Léon (début avril)», en Durkheim (1975, 2: 463-464).

- (1901): «Lettre au Directeur de la Revue philosophique», Revue philosophique, 52: 704. [Repr. en Durkheim (1975), 1: 52-53.]

- (1905): «La sociologie», en Ministère de l'Instruction publique et des Beaux-Arts, La science française, París: Larousse, 1: 39-49. [Repr. en Durkheim (1975), 1: 109-118.]

- (1975): Textes, París: Minuit, 3 vols.

Hauser, H. (1903): L'enseignement des sciences sociales, París: Marescq.

LAPIE, P. (1895): «L'année sociologique 1894», Revue de métaphysique et de morale, 3: 308-339.

LEgOYT, A. (1881): Le suicide ancien et moderne, París: Drouin.

LÉVY-BRuHL, L. (1895): "Questions sociologiques», La revue politique et littéraire. Revue Bleue, 4e s., 3: 776-782.

LUKES, S. (1973): Emile Durkheim. His life and work, Londres: Allen Lane.

MAuss, M. (1925): «In memoriam. L'oeuvre inédite de Durkheim et de ses collaborateurs», L'Année sociologique, n. s., 1: 7-29. [Repr. en Mauss (1969), 3: 473-499.]

- (1969): Oeuvres, París: Minuit, 3 vols.

- (1979): «L'oeuvre de Mauss par lui-même», Revue française de sociologie, 20: 209-220.

Merllie, D. (1989): «Lévy-Bruhl et Durkheim», Revue philosophique, 114: 439-514.

Milet, J. (1970): Gabriel Tarde et la philosophie de l'histoire, París: Vrin.

MORSELLI, E. (1879): Il suicidio, Milano: Dumolard. 
Pareto, V. (1898): «C. r. de E. Durkheim, Le suicide», Zeitschrift für Sozialwissenschaft, 1: 7880. [Repr. en Pareto (1966): 122-124.]

- (1966): Mythes et idéologies (Oeuvres complètes, VI), Genève-Paris: Droz.

Paulhan, J., y TARde, Guillaume (de) (1980): Correspondance (1904-1920), París: Gallimard.

POURnIN, M. (1904): "La sociologie et les sciences sociales», Revue internationale de sociologie, 12: 83-89, 161-167.

Sorel, G. (1895): "Théories pénales de MM. Durkheim et Tarde», Archivo di psichiatria, cienze penali ed antropologia criminale, 16: 219-228.

TARDE, G. (1886): La criminalité comparée, París: Alcan.

- (1891): «Note sur la statistique criminelle de 1888», Archives d'anthropologie criminelle, 6: 325-330.

- (1893): "Questions sociales», Revue philosophique, 35: 618-638. [Repr. en Tarde (1895c): 175-209.]

- (1894): «Les délits impoursuivis», Archives d'anthropologie criminelle, 9: 641-650. [Repr. en Tarde (1895c): 211-222.]

- (1895a): La logique sociale, París: Alcan.

- (1895b): «Criminalité et santé sociale», Revue philosophique, 39: 148-162. [Repr. en Tarde (1898): 136-158.]

- (1895c): Essais et mélanges sociologiques, Lyon: Storck (París: Masson).

- (1895d): «La sociologie élementaire», Annales de l'Institut internationale de sociologie, 1: 209242. [Repr. como "Les deux éléments de la sociologie» en Tarde (1898): 63-94.]

- (1896a): «L'idée de l'“organisme social”", Revue philosophique, 41: 637-646. [Repr. en Tarde (1898): 120-135.]

- (1896b): "La criminalidad profesional», Archives d'anthropologie criminelle, 11: 538-560. [Repr. en Tarde (1898): 162-194.]

- (1898): Etudes de psychologie sociale, París: Giard \& Brière.

- (1901): «La réalité sociale», Revue philosophique, 52: 457-477.

- (1973): Ecrits de psychologie sociale, Toulouse: Privat.

TARDE, Guillaume (de) (1904): «Lettre à Jean Paulhan (été)», en Paulhan y Tarde, Guillaume (de) (1980: 19-21).

TOURnier, C. (1898): «C. r. de E. Durkheim, Le suicide», Archives d'anthropologie criminelle, 13: $326-335$.

(Traducción: Amparo LASÉN.)

\begin{abstract}
Durkheim obtained some of Suicide's stastistical data from Tarde, Director of Statistics at the French Justice Ministry from 1894 to 1904, whose theoretical positions were deeply criticized in Suicide. This article is a research on the Durkheim-Tarde relationships during the writing of Suicide. It reconstructs its composition calendar and analyses durkheimian strategy in orden to obtain the data Durkheim needed from someone who was both his rival in the rising sociology and object of his criticism. It also furnishes some Durkheims unpublished letters to his rival, in an appendix.
\end{abstract}

